\title{
Dinámicas de la actividad científica del Instituto de Investigaciones Socio-Económicas de la Universidad Nacional de San Juan de Argentina
}

\author{
Gonzalo Miguel Castillo ${ }^{1}$
} Flavia Cecilia Prado ${ }^{2}$

\section{Resumen}

Este artículo fue un estudio de caso en torno a las capacidades de investigación generadas en el Instituto de Investigaciones Socio-Económicas de la Facultad de Ciencias Sociales, perteneciente a la Universidad Nacional de San Juan, en Argentina. Aborda los principales acontecimientos históricos de la institución y su dinámica actual, tuvo en cuenta las áreas de conocimiento en las cuales se desarrollan sus investigaciones. A su vez, este trabajo ha descrito las dinámicas de la revista académica producida en este instituto, denominada REVIISE.

La metodología fue un abordaje objetivo/descriptivo. Se procedió a analizar el papel, devenir y accionar del IISE y las modalidades de proyectos de investigación ejecutados. Fueron indagados el funcionamiento de la REVIISE, identificando perfiles académicos de sus autores, circuitos de difusión y redes académicas; y la conformación de comités y consejos editoriales y académicos que componen esta publicación. Se propone realizar un recorrido que atraviesa lo institucional, los agentes académicos y la principal red de difusión de la producción de conocimiento para comprender las capacidades investigativas del Instituto de Investigaciones Socio Económicas.

Palabras clave: Circuitos de difusión universitarios; Instituto de Investigaciones Socioeconómicas; Trayectorias académicas.

\section{Summary}

This article was a case study about the research capabilities generated in the Social Sciences Faculty at the Socio-Economic Research Institute, belonging to the National University of San Juan. It addresses the main historical events of the institution and its current dynamics, the areas of knowledge in which its research is carried out were taken into account. Sequentially, this work has described the dynamics of the academic journal produced in this institute, denominated REVIISE.

1 Investigador del Instituto de Investigaciones Socioeconómicas, FACSO, Correo: castigonzalo@gmail.com

2 Investigadora del Instituto de Investigaciones Socioeconómicas, FACSO. Correo: flaviacprado@gmail.com

Recibido: 05/10/2017 Aprobado: 23/11/2017 
The methodology was an objective / descriptive approach. Through this proposal, it was proceeded to analyze the role, evolution and actions of the IISE and the modalities of research projects carried out in it. The functioning of the REVIISE was investigated, identifying academic profiles of its authors, dissemination circuits and academic networks; and the conformation of Committees and Editorial and Academic Councils that compose this publication. It is proposed to make a journey through the institutional, academic agents and the main network of dissemination of knowledge production to understand the research capabilities of the Socio-Economic Research Institute.

Key Words: University dissemination circuits; Socioeconomic Research Institute; Academic trajectories.

\section{Introducción}

El artículo es una descripción panorámica que aborda aspectos históricos contextuales del Instituto de Investigaciones Socio-Económicas (IISE) de Argentina, su composición del equipo de mujeres y hombres investigadores, y áreas de conocimiento donde ejecutan sus proyectos de investigación. A su vez, se realiza una lectura descriptiva acerca de su aparato de difusión académico: Revista de Ciencias Sociales y Humanas (REVIISE); indagando sus lógicas de funcionamiento, al identificar la constitución de su comité editorial, consejo académico, autores y temáticas que participan en el proceso de publicación.

De esta manera el objetivo planteado consiste en realizar un recorrido que atraviesa lo institucional, los agentes académicos y caracterizar una de las aristas del proceso de difusión del conocimiento (REVIISE). Es pertinente aclarar que existe escasa información sin sistematizar e investigaciones acerca de la temática en la provincia de San Juan en Argentina. Por ello, la presente comunicación es un estudio de tipo descriptivo, cuyas conclusiones pretenden posicionarse como aportes a una línea de investigación referente a las capacidades de investigación de la Universidad Nacional de San Juan (UNSJ) que se encuentra en proceso de gestación.

\section{Desarrollo}

\section{La Universidad como un complejo agente en el circuito de Ciencia y Técnica}

En el ámbito académico, las universidades enfatizan en la investigación y en las publicaciones como medio para el reconocimiento y el ascenso en la escala académica; asimismo dan prioridad, mediante el ejercicio de políticas específicas, a ciertas áreas de la ciencia y la tecnología. Dado que las universidades cuentan con la infraestructura básica para la investigación, ellas pueden ser una fuente de asistencia para la investigación y el desarrollo. "Las universidades desempeñan un papel fundamental en la 
investigación científica de cualquier país. Ellas son, en primer lugar, instituciones existentes que cuentan con bibliotecas, laboratorios y personal altamente capacitado. Tienen una orientación hacia la investigación y una tradición que la avala". (Altbach, 2009, p. 329).

Por ello, las instituciones científicas son organizaciones específicas, con entramados organizativos complejos; procesos y metodologías enfocadas hacia la apropiación, producción y transmisión de un producto especial: el conocimiento científico. La universidad es la institución paradigmática donde se produce conocimiento científico a través de la investigación que se desarrolla e institucionaliza prioritariamente a través de los llamados grupos o equipos de investigación.

La investigación científica, requiere de un esfuerzo compartido, pero no sólo como consecuencia de una política interna de la organización, sino sobre todo, como fundamento de la institucionalidad de la ciencia, la cual se desarrolla en el interior de una comunidad de investigadores, que puede lograr la consolidación de un proyecto de investigación y de conocimiento de largo plazo, y asegure una continuidad en las líneas de trabajo, así como la acumulación de resultados significativos. "Teniendo en cuenta la importancia que tienen los grupos de investigadores como eslabón fundamental en la producción del conocimiento científico, se hace necesario conocer cuáles son las trayectorias de los profesionales que se dedican a la tarea de investigar" (Medina, 2000, p. 385). Este autor señala como parte de la construcción de la trayectoria profesional, intensas negociaciones y luchas.

Los agentes logran ocupar determinadas posiciones hacia el interior de un campo, en palabras de Bourdieu (2008), en base a las diferentes cuotas de capital que han logrado agenciar como propias. De la misma forma, existen posiciones que tienen como función controlar la incorporación de los nuevos agentes al campo y hay quienes luchan por incorporarse y mantenerse dentro del mismo. La formación no sólo incorpora a un campo de conocimiento, que contribuye en legitimar el ejercicio de una práctica, sino que se convierte en la base para formar a otros, de tal suerte que el apropiarse de un campo de conocimiento especializado se convierte en el legado o herencia para los investigadores en formación.

En la trayectoria profesional los investigadores han pasado por un proceso escolarizado que les proporcionó un conocimiento especializado para la práctica. La formación en determinadas instituciones con determinados actores proporciona a los investigadores la incursión en un saber especializado en diversos niveles y ámbitos, por ejemplo el teórico epistemológico y el metodológico, que implica múltiples formas de establecer relaciones con el campo de conocimiento adscrito, por ejemplo, como lo hemos dicho con conocimientos especializados, con prácticas sobre la investigación, con sentidos sobre el ejercicio de una práctica (Medina, 2000). 


\section{EDUCACIÓN}

Otro elemento constitutivo del hacer científico es la ampliación y difusión del conocimiento que se construye, las instancias de producción, circulación, difusión y recepción de los discursos académicos suelen compartir características estructurales con los modos de producción de contenidos mediáticos. Es pertinente entender que las publicaciones científicas poseen ciertos patrones particularmente endógenos inherentes a su constitución, aunque existen y se manifiestan en ellas lógicas generales a todo proceso de emisión/recepción de contenidos mediatizados. En palabras de Hall (2013) el proceso comunicacional se entiende como una estructura que es "producida y sostenida a través de la articulación de momentos relacionados pero distintivos -Producción, Circulación, Distribución/Consumo, Reproducción-." (p. 203) y a su vez, como una "estructura compleja dominante», sostenida a través de la articulación de prácticas conectadas, cada una de las cuales, retiene, sin embargo, su carácter distintivo y tiene su modalidad específica [...]. (Hall, 2013, p. 203).

Cada instancia que compone el proceso científico de comunicación gozaría de cierta autonomía relativa, aunque siempre se encuentran supeditadas al momento de codificación inicial. Por otra parte, es necesario detenerse en reflexionar acerca de cómo las instituciones que publicitan discursos, son atravesadas por diferentes componentes que complejizan el acto de difusión, como el caso de las "estructuras institucionales y redes de producción, sus rutinas organizacionales e infraestructuras técnicas" (Hall, 2013, p. 221). Paralelamente, el proceso de producción de discursos también es atravesado por diversas construcciones de significados e ideas como pueden ser "el conocimiento operativo de las rutinas de producción, habilidades técnicas, ideologías profesionales, conocimiento institucional, definiciones y prejuicios e ideas preconcebidas sobre la audiencia" (Hall, 2013, p. 221).

Estas subjetivaciones que acontecen al interior de las instituciones que producen procesos comunicativos presentan algunos aspectos correlativos a los campos en los cuales se insertan. En El Oficio del Científico (2003), Bourdieu y Passeron presentan una de las definiciones más completas acerca del "campo científico". Se trata de un espacio de fuerzas dotado de una estructura y a la vez un espacio de luchas para conservar o transformar esa relación de fuerzas. En este sentido, afirma que: “(...) son los agentes, es decir, los científicos aislados, los equipos o los laboratorios, definidos por el volumen y la estructura del capital específico que poseen, quienes determinan la estructura del campo que los determina, es decir, el estado de las fuerzas que se ejercen sobre la producción científica, sobre las prácticas de los científicos. El peso asociado a un agente, que soporta el campo al mismo tiempo que contribuye a estructurarlo, depende de todos los restantes agentes, de todos los restantes puntos del espacio y de las relaciones entre todos los puntos, es decir, de todo el espacio" (Bourdieu et al., 2003, p. 65).

Cada agente se caracteriza por su trayectoria social y su posición en el campo y un campo posee autonomía relativa; las luchas que en él se desarrollan tienen una 
lógica interna, pero también el resultado de las luchas (económicas, sociales, políticas) externas al campo pesa fuertemente sobre el efecto de las relaciones de fuerza internas. A su vez, como también explicita Bourdieu, "el capital universitario se obtiene y se mantiene a través de la ocupación de posiciones que permiten dominar a otras posiciones y sus ocupantes" (Bourdieu, 2008, p.14).

El objetivo de este trabajo, como se ha mencionado en momentos anteriores, no constituye la lectura de un campo científico que aborde todo un espectro nacional. Sino es un estudio de caso que tiene su anclaje en un fragmento del circuito académico de la provincia de San Juan, como es el IISE y sus capacidades de investigación, es decir sus recursos humanos y la revista REVIISE. Sin embargo, este instituto de investigaciones no es ajeno a las lógicas de producción y difusión del conocimiento científico que se configura en América Latina. Por lo tanto, es un recorrido pertinente para su descripción, recurrir a herramientas teóricas propuestas por Beigel (2016) respecto a las nuevas formas de construcción del prestigio académico en torno a la publicación y la denominada dependencia intelectual.

El conflicto de la dependencia académica es conceptualizado por Beigel como "una estructura desigual de producción y circulación del conocimiento, construida históricamente desde los tradicionales "centros de excelencia", sin la participación de las comunidades científicas periféricas"; en la cual, "las publicaciones científicas jugaron en este proceso un papel determinante." (Beigel, 2016, p. 9). Por lo tanto, toman un carácter descollante al momento en que un académico toma posición respecto a su hacer publicación, tensionando entre lo que puede entender como localías y/o transnacionalidades, y las lógicas de legitimación que invisten cada una de ellas. Esta dicotomía lleva a diversos intelectuales como los que componen la comunidad científica de Argentina y dirimir entre publicar en sectores periféricos, pensándose ajenos a las directrices temáticas transnacionales. $\mathrm{O}$ adecuarse a las agendas internacionales y escindiéndose del ámbito local (Beigel, 2016).

Esta constante tensión entre centro y periferia también plasmada en la agenda temática de los procesos comunicativos de discursos científicos colocan en una encrucijada al investigador latinoamericano, que pugna entre investigar y producir elementos de difusión acordes a las realidades contextuales en las que se desempeña, o por el contrario tratar de ganar otras cuotas de prestigio transnacionalizado su hacer académico. En ese caso es importante mencionar una de las categorías que Beigel (2016) retoma de Atalas (2014) en la cual queda de manifiesto como investigadores periféricos llevan a cabo grandes esfuerzos para lograr cumplir protocolos específicos y ser rankeados internacionalmente. Esto ocurre tanto como investigadores en casos individuales, como en publicaciones académicas con sus intentos de lograr una indexación internacional. 


\section{EDUCACIÓN}

Contexto histórico-social del origen de la Universidad Nacional de San Juan

La Universidad Nacional de San Juan es una institución universitaria dedicada a la enseñanza, investigación, creación y difusión del saber en todos sus órdenes (científico, técnico, filosófico y artístico) y a la formación integral de profesionales al servicio del bien común (Ord. $\mathrm{N}^{\circ}$ 5-98-AU). Se crea sobre la base de las instituciones de nivel superior preexistentes en la provincia de San Juan, de la República Argentina, las cuales comprendían diferentes jerarquías institucionales:

1. La Universidad Provincial "Domingo Faustino Sarmiento".

2. El Instituto Nacional del Profesorado Secundario.

3. La Facultad de Ingeniería, Ciencias Exactas, Física y Naturales, dependiente de la Universidad Nacional de Cuyo.

Desde su creación, hasta la actualidad, la UNSJ ha sufrido diferentes reorganizaciones, algunas parten de su propia dinámica y otras vinculadas a los procesos sociohistóricos que ha atravesado, tales como los procesos de interrupción democrática, llegando a la actualidad a ser constituida por cinco facultades: Ingeniería; Ciencias Exactas, Físicas y Naturales; Filosofía, Humanidades y Artes; Arquitectura, Urbanismo y Diseño y Ciencias Sociales. La UNSJ fue fundada en 1973, período que a nivel nacional involucra sucesivos cambios políticos, económicos, sociales y culturales. Argentina venía de un régimen político dictatorial, que luego fue democráticamente sustituido por las elecciones presidenciales de marzo de 1973 que dieron como triunfador a Héctor Cámpora; quien tres años más tarde sería depuesto por el último y más cruel Golpes de Estado.

Su planificación originaria tuvo lugar dentro de la segunda expansión del Sistema Universitario Argentino ejercido durante la dictadura militar de 1966. En relación con la educación superior, durante los gobiernos de facto de Onganía y Lanusse se limitó el ingreso y comenzó a aplicar el plan de "Creación de Nuevas Universidades Nacionales" elaborado por Alberto Taquini. Este consistía en crear universidades pequeñas repartidas en la geografía nacional con el objeto de dispersar la población estudiantil que se encontraba en proceso de masificación, como son los casos de la Universidad de la Plata (UNLP), La Universidad de Buenos Aires (UBA), Universidad Nacional de Cuyo (UNCU), Universidad Nacional de Córdoba (UNC) y la Universidad Nacional de Rosario (UNR).

El Plan Taquini, considerado hasta la actualidad por el campo académico argentino, al menos controversial, era argumentado por sus ejecutores y mentores como un paso necesario para la federalización de la educación superior en Argentina. "El Dr. Taquini afirmaba que gran parte de la juventud abandonaba su provincia natal, para trasladarse a las grandes ciudades donde proseguían sus estudios [...] lo que negativamente en el progreso regional de muchas zonas del interior del país [...]" (UNSJ, 1994, p. 35). 
Sin embargo, la desagregación de los grandes centros universitarios y la creación de estas nuevas universidades ediliciamente separadas en las diversas regiones del país dificultaron los procesos de reunión, comunicación y manifestación por sus derechos de los estudiantes universitarios de Argentina.

La UNSJ no estaba prevista en el listado inicial de creación de nuevas universidades, sin embargo, en la provincia se había conformado un grupo de académicos que bregaba en pos de la creación de una Universidad Nacional desde la creación de la Universidad Provincial la que sumara su accionar bajo el mismo objetivo ya que, entre otros motivos, el Gobierno Provincial encontraba serios inconvenientes para sostener presupuestariamente esta institución. Este fuerte movimiento social consiguió que se realizara un estudio de factibilidad para la instauración de la UNSJ y su creación se concretó mediante ley 20.367, el día 10 de Mayo de 1973 (UNSJ, 2013).

Mediante este contexto, es posible sostener que:

Desde finales de los años '6o y comienzos de los '7o se produjo el fenómeno de la multiplicación de núcleos de militancia juvenil universitaria, siempre enmarcados en un contexto de fuertes luchas y debates contra el poder dictatorial. La "resistencia a la dictadura" y la "resistencia a la represión" eran por aquellos años, la consigna social que regía el creciente activismo estudiantil en los claustros universitarios sanjuaninos (Algañaraz, 2014, p. 18).

Desde el mismo momento de su génesis en 1973, se abrió hacia el interior de la UNSJ una etapa de numerosos debates y enfrentamientos entre los distintos agentes y sectores ligados a ella. El proceso de creación de la UNSJ se vivió con intensidad en medio de un contexto socio-político marcado por la restauración democrática. El 23 de junio de 1973, se nombró al ingeniero Julio Rodolfo Millán como rector normalizador de la universidad, iniciándose a nivel provincial -al igual que en todo el campo académico nacional- un proceso de reconstrucción universitaria, caracterizado por el advenimiento y consolidación de la Juventud Universitaria Peronista (J.U.P.).

Sin embrago, esta situación se ve fuertemente afectada en la década de 1976. La dictadura militar de 1976 asume características inusuales, no sólo por el grado inhumano que adquirió la represión para implantar el terrorismo de Estado, sino también por la imposición de un cambio de modelo económico en el país. Esta modificación del patrón de acumulación capitalista se tradujo en una etapa de marcada hiper-concentración de poder y riqueza en manos de la nueva alianza de poder: la aristocracia financiera.

En la Universidad hubo docentes que fueron cesanteados de sus cátedras por cuestionamientos ideológicos; "esta fue una época que se caracterizó por la intolerancia de las ideas y a consecuencia de éstos, fueron separados de sus cargos en la universidad a través de expresas resoluciones, docentes, investigadores y no docentes incluidos 


\section{EDUCACIÓN}

en las tan mentadas "listas negras"[...]" (UNSJ, 1994, p. 56). Siendo así, un período en el cual la autonomía universitaria se vio completamente avasallada (UNSJ, 1994).

Durante estos años fue modificado el Reglamento Académico, se redujeron los períodos destinados a la toma de exámenes, las condiciones para obtener la regularidad se volvieron más exigentes y se establecieron cupos de ingreso y exámenes de admisión. El régimen de censura y el temor impuesto por la dictadura produjeron, en poco tiempo, la desmovilización total de los docentes, estudiantes y personal de apoyo universitario. Las asambleas, las discusiones, los debates, es decir, el ejercicio de toda práctica que permitía el desarrollo de actividades críticas, desaparecieron de los claustros universitarios. El silencio impuesto por el miedo daba cuenta que la libertad de pensamiento, de expresión y de reunión habían sido coartadas (Algañaraz, 2014).

Se revisaron y rediseñaron los planes de estudio existentes y fueron suprimidas algunas materias consideradas peligrosas en el nivel de carreras de grado. En el comienzo de este período se vivió una experiencia singular y devastadora en la biblioteca de la Facultad. Militares y fuerzas de seguridad procedieron al secuestro de todo el material bibliográfico considerado de carácter subversivo. En este operativo, fueron secuestrados numerosos volúmenes provocando un menoscabo del patrimonio cultural de nuestra casa de estudios.

A nivel nacional, se dictaron decretos y resoluciones ministeriales por las que se prohibía la tenencia y circulación de determinadas obras consideradas ideológicamente peligrosas, como la bibliografía marxista y la vinculada con la liberación de los países del tercer mundo. Esta situación se extendió hasta diciembre de 1983, cuando el país retornaba a la democracia; libros y obras de autores proscriptos volvían a ocupar un lugar en las bibliotecas.

Una medida de extrema gravedad para las Ciencias Sociales fue el cierre de la carrera de Sociología. Por el período de dos años, se suspendió la inscripción en la mencionada carrera, medida tomada como forma de desactivar por la fuerza todo pensamiento o sistemas de ideas que cuestionaran ideológicamente el régimen militar. Otras carreras de nuestra Facultad se vieron amenazadas de supresión, pero, finalmente, no sucedió (Algañaraz, 2014).

Con el advenimiento de la democracia en 1983, se planteó como objetivo "un proceso de intervención, normalización y democratización de las distintas instituciones del sector [científico académico]. [...] Las universidades nacionales y el CONICET fueron intervenidos, con la intención de normalizarlos." (Albornoz y Gordon, 2011, p. 12). Estas intervenciones de diferentes instituciones científicas y académicas fueron argumentadas en el hecho que las autoridades que las regían habían sido nombradas por el gobierno de facto de la última dictadura cívico militar. 
La crisis económica disparada durante el gobierno de Alfonsín, lo llevó a finalizar su mandato anticipadamente. Se llamó a elecciones presidenciales en 1989, resultando ganador Carlos Menem con un proyecto político de corte neoliberal. Siguiendo a Albornoz y Gordon (2011), existieron dos facetas en la política científica que caracterizaron a los dos mandatos presidenciales de Menem.

Una primera denominada por los autores, mencionados más arriba, como "reacción tradicionalista" (Albornoz y Gordón, 2011, p. 16), en la cual "volvieron a ocupar un lugar destacado en la conducción de las instituciones científicas algunos investigadores que habían estado ligados a las intervenciones en tiempos de las dictaduras militares" (Albornoz y Gordón, 2011, p. 16). Y una segunda llamada "modernización tecnocrática" (Albornoz y Gordón, 2011, p. 16) donde "se impulsaron medidas tendientes a dotar a los organismos públicos de criterios de gestión empresarial, del mismo modo que se intentó alinear a las políticas de ciencia y tecnología según el objetivo final de la innovación" (Albornoz y Gordón, 2011, p. 26). Sin embargo, como Albornoz y Gordon (2011) sostienen, el concepto de innovación de utilizado de forma acrítica, pudiéndolo entender como un significante vacío.

En la UNSJ, el primer estatuto propio fue aprobado en 1989 y modificado en el año 2001. En él se fijaron los objetivos institucionales, las funciones académicas y las formas de organización y gobierno universitario entendiendo que la elección de sus autoridades "se realiza mediante voto directo ponderado de cada uno de los claustros, conservando el gobierno cuatripartito, y siendo en términos generales las representaciones estamentales del 50\% para docentes, 25\% para estudiantes, $12,5 \%$ para egresados y 12,5\% para el personal de apoyo universitario"(UNSJ, 2013, p. 152).

El órgano máximo de conducción lo constituye la Asamblea Universitaria, representante de la autonomía universitaria. Cada sector elige democráticamente a sus representantes, los que actúan en los distintos órganos de gobierno de la Universidad:
A. Asamblea Universitaria.
B. Consejo Superior
C. Consejos Directivos -uno por cada facultad- .

A.La Asamblea Universitaria es el máximo órgano de gobierno de la Universidad. Está integrada por el Rector, todos los miembros del Consejo Superior y los integrantes de los Consejos Directivos de todas las Facultades. Este cuerpo colegiado, numeroso, tiene una sesión ordinaria anual. La asamblea en pleno es quien elabora y aprueba el Estatuto de la Universidad y todas sus reformas, también puede crear y suprimir facultades, teniendo todos sus miembros voz y 


\section{EDUCACIÓN}

voto. Los representantes estudiantiles y graduados, se renuevan todos los años. Los representantes docentes y de apoyo duran cuatro años en su función.

B. El Consejo Superior es el órgano ejecutivo de gobierno universitario. Es presidido por el Rector e integrado por los decanos de las cinco facultades, además de representantes de los docentes, los egresados, los alumnos, el personal de apoyo y representantes de los Colegios Pre-universitarios. Este órgano tiene a su cargo las más importantes decisiones a nivel de toda la Universidad.

C. Los Consejos directivos son los órganos de gobierno de las facultades. Así cada una de las cinco facultades de la UNSJ posee un Consejo Directivo, integrado por el Decano de esa facultad, representantes de los claustros docentes, alumnos, egresados y personal de apoyo. Todos estos representantes son elegidos por sus pares en comicios democráticos. Las decisiones más importantes de una facultad, desde la creación de carreras nuevas hasta la aprobación del presupuesto, pasan por este Consejo.

En el contexto sociopolítico nacional es importante destacar que durante 2001 y 2002 "la crisis económica, financiera, social, política e institucional [...] dejó como corolario la cesación del pago de deuda soberana más grande de la historia. [...] Desde 2002 comenzó una paulatina recuperación económica que alivió las cuentas públicas." (Albornoz y Gordon, 2011, p. 28). En el año 2003 gana las elecciones presidenciales Néstor Kirchner y nombró como Secretario de Ciencia, Tecnología e Innovación Productiva a Tulio del Bono, quien "mostró un talante dialoguista y aportó como novedad el propósito de retomar la elaboración de planes estratégicos de medio y largo plazo" (Albornoz y Gordon, 2011, p. 29). Algunos de sus objetivos fueron "Creación y aplicación de conocimiento para la explotación responsable de los recursos naturales, protegiendo el ambiente." Y "Aumento de la base científica y de la capacidad tecnológica" (Albornoz y Gordon, 2011, p. 31).

En el 2007, durante la primera presidencia de Cristina Fernández se creó el Ministerio de Ciencia, Tecnología e Innovación Productiva (MINCYT). "Esta medida, que en la práctica conlleva una separación entre la política universitaria y la de investigación fue celebrada públicamente como una mayoría de edad de la política científica y un reconocimiento largamente esperado a la importancia política de la ciencia" (Albornoz y Gordon, 2011, p. 40).

Para el 2011 la UNSJ estaba compuesta por 65 carreras de grado y 30 de posgrado. Ellas contienen un total de 19.530 estudiantes y 2.757 docentes en ejercicio. Hay que mencionar, por otro lado, que la investigación tiene un papel importante dentro de la universidad. Así, en el 2011 se estaban desarrollando 350 Proyectos de Investigación financiados por la SECyT-UNSJ. Había en ejecución 55 Proyectos de Jóvenes Investigadores (PROJOVI), financiados en convenio con la SECITI-Gobierno de San Juan. 
La génesis del Instituto de Investigaciones Socio- Económicas (IISE) en el ámbito de la Facultad de Ciencias Sociales(FACSO)

La FACSO sienta sus bases en la Facultad de Humanidades de la Universidad Provincial Sarmiento y en la Escuela de Periodismo (EP), que había nacido en la década de 1950. En 1961 la EP había aprobado el plan para su nueva carrera de Ciencias Políticas y Sociales, orígenes de los departamentos de Ciencias Políticas y Sociología de la actual FACSO. Durante la década de 1970, la EP pasó a denominarse Departamento de Ciencias de la Comunicación, en un contexto en que la Facultad de Humanidades de la Universidad Sarmiento ya tenía carreras de grado como Licenciatura en Ciencias de la Comunicación, Ciencias Políticas, Administración de Empresas, y estudios Superiores en Servicio Social (UNSJ, 2013, p. 6).

El período 1968-1973 fue una etapa de gran movilización para los claustros universitarios. Existía una importante puja estudiantil y docente en favor de una vida democrática, en tiempos de la Dictadura de Onganía. En medio de esto, la Universidad Provincial Sarmiento obtuvo reconocimiento nacional de sus títulos y, poco más tarde, fue creada la UNSJ. Finalmente, en 1974 la FACSO se incorporó a la Universidad Nacional.

Durante la última dictadura cívico-militar, en materia de investigación, se atravesaron difíciles momentos, sin embargo, a pesar de las dificultades, limitaciones ideológicas y presupuestarias se realizaron durante esta época una serie de trabajos significativos que fueron publicados en pequeñas tiradas, permitiendo una limitada divulgación y transferencia al medio. A su vez, como sostiene Algañaraz "el amplio grupo de carreras que se dictaban en la FACSO se vieron seriamente amenazadas de supresión, esta medida de extrema gravedad se concretó únicamente en Sociología, que entre 1979 y 1980 tuvo suspendida la incorporación de nuevos alumnos" (UNSJ, 2013, p. 250).

En 1982 se creó el Centro de Estudios para la Integración Latinoamericana (CEILA), cuyo objetivo central era contribuir a la integración social, cultural, económica y política de América Latina. También en ese mismo año se creó el Instituto de Investigaciones Administrativas Contables (IAC). Este espacio, que en un comienzo estaba integrando el Instituto de Investigaciones Socio Económicas, fue separado a fin de darle una mayor autonomía e impulsar más intensamente las investigaciones relacionadas con los aspectos administrativos y contables de la gestión empresarial.

La FACSO es una de las cinco facultades que integra la UNSJ. Cuenta con seis Departamentos Académicos de estudios de grado (Ciencias de la Comunicación, Ciencias Políticas, Ciencias Económicas, Ciencias Jurídicas, Sociología y Trabajo Social), cuatro de posgrado (Especialización en Gestión y Vinculación Tecnológica, Maestría en Metodología de la Investigación en Ciencias Sociales, Maestría en Políticas Sociales, 


\section{EDUCACIÓN}

y Doctorado en Ciencias Sociales) y dos institutos de investigaciones (Instituto de Investigaciones Socio Económicas e Instituto de Investigaciones Administrativas y Contables). Asimismo, con el propósito de incentivar las actividades de investigación y de alcanzar una mayor articulación entre las actividades docentes y de investigación se crearon en cada departamento gabinetes de investigación:(GEICPO -Gabinete de Estudios e Investigaciones en Ciencias Políticas-, GEICOM-Gabinete de Estudios e Investigaciones en Ciencias de la Comunicación-. GEITS-Gabinete de Estudios e Investigaciones en Trabajo Social-, GEIS -Gabinete de Estudios e Investigaciones en Sociología- GEICJU --Gabinete de Estudios e Investigaciones en Ciencias JurídicasGEIA -Gabinete de Estudios e Investigaciones en Administración-.

En 1974 se crea el Instituto de Investigaciones Socio-Económicas (IISE), luego de varios intentos de concretar centros e Institutos de Investigación en la facultad. Los objetivos originarios planteados para este instituto consistían en obtener un diagnóstico socioeconómico de la Provincia de San Juan. Además, la ampliación, sistematización y difusión de conocimientos socioeconómicos y administrativos contables tendientes a una mejor y mayor comprensión de la situación provincial, regional y nacional. También encuadra en sus objetivos la realización de cursos, ciclos de conferencias, seminarios y todo tipo de actividad que tienda al perfeccionamiento, capacitación y actualización de los docentes, investigadores y profesionales (UNSJ,1994, p. 129).

En este sentido, se catalogaban como prioritarias aquellas investigaciones con utilidad social tangible y cuyos resultados sean transferibles a organismos públicos y organizaciones sociales privadas y que signifiquen un aporte original para la solución de problemas.

En Abril del año 1982 se creó el Instituto de Investigaciones Administrativas Contables (IAC). "Esta área, que en un comienzo estaba integrando el Instituto de Investigaciones Socioeconómicas, fue separada a fin de darle una mayor autonomía e impulsar más intensamente las investigaciones relacionadas con los aspectos administrativos y contables de la gestión empresarial" (UNSJ, 2013, p. 6).

Desde la llegada a la democracia en 1983 hasta los primeros años de la década de 1990 constituyó un período de crecimiento en materia investigativa para el IISE. "Se abrieron mejores perspectivas al tener el claustro de profesores una cierta estabilidad. Los que habían concursado tenían un período mínimo de siete años y a los no concursados se les renovaron sus designaciones [...]" (UNSJ, 1994, p.142). Al ser docentes investigadores, los miembros del IISE, transferían de manera casi simultánea sus hallazgos a las cátedras que dictaban (UNSJ, 1994), construyendo de esa forma una ágil articulación entre la investigación, docencia y difusión de sus investigaciones.

Actualmente, el IISE ejecuta además de tareas de investigación, tareas de formación en materia de post-grados. En este instituto se desarrolla una Maestría en 
Metodología de la investigación en Ciencias Sociales y un Doctorado en Ciencias Sociales. De la misma forma, edita una revista académica denominada REVIISE, con lo cual abarca actividades de formación en la investigación y divulgación del conocimiento científico construido.

Paralelamente, la acción investigativa que desarrolla el IISE en el estudio de la problemática de la sociedad sanjuanina es amplia y mantiene en su seno un importante número de investigadores que se dedican a la docencia. Ellos se reparten entre las grandes áreas de investigación: salud y sociedad; género y sociedad; estado, gobierno y sociedad; comunicación y sociedad; educación, cultura y sociedad; socioeconómica regional: rural y urbana; Ambiental y Demografía.

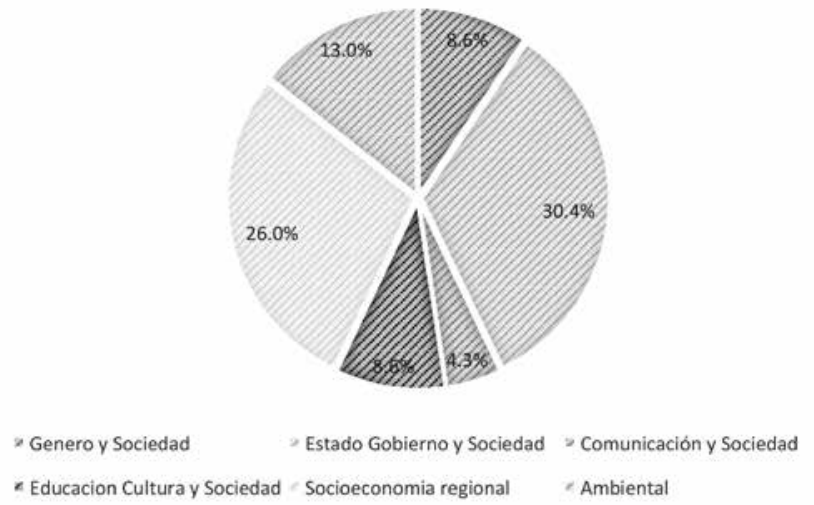

Figura 1: Proyectos ejecutados según área de investigación 2008-2010

Como se puede observar, en el periodo 2008-2010 las áreas de investigación que tienen mayor representación son aquellas relacionadas con temas sobre Estado; Gobierno y Sociedad; $(30,4 \%)$ y Socio-economía regional $(26 \%)$. Dichas áreas de investigación nuclean proyectos, que son presentados por los docentes investigadores según los temas que corresponden a cada una. Estas áreas no son excluyentes, en el caso que surjan proyectos que aborden otras temáticas distintas a las mencionadas, siempre y cuando estén dentro del ámbito socioeconómico. La mayoría de los proyectos son bianuales.

Con respecto a la estructura organizacional del IISE, se constituye por un Director y Subdirector; y un Consejo conformado por tres investigadores que revisten el carácter de titulares y tres suplentes. Los mismos son elegidos cada cuatro años y por la totalidad de investigadores-docentes que integran el instituto. Ello permite una autoridad parcialmente delegada y distribuida en los niveles de supervisión, situados en niveles jerárquicos inferiores.

Asimismo, es relevante conocer la composición del cuerpo de docentes- investigadores que se desempeñan en el IISE. Tal y como se observa en la figura 2, se puede 
decir, tomando como base los proyectos ejecutados en el periodo 20011-2012, que dentro de las diferentes áreas de investigación existe un total de 125 investigadores de los cuales 95 son mujeres y 30 son varones.

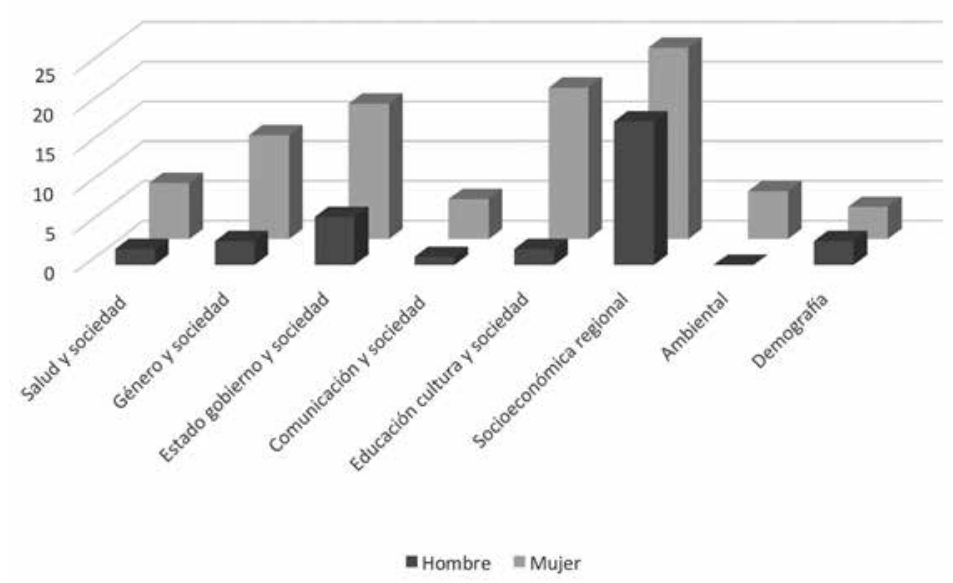

Figura 2: Investigadores del IISE según género y área de investigación

En cuanto a la formación e incentivos a la investigación, la UNSJ cuenta con un sistema de becas destinada a los egresados, los programas de becas de investigación son, además de los subsidios, el otro pilar de las políticas científicas. Como es conocido, éstas consisten en la asignación de un estipendio para la realización de una investigación específica, usualmente asociada a estudios de posgrado.

Según Vasen (2013), algunas de las becas que generalmente son implementadas por la mayoría de las universidades nacionales son las siguientes:

- De grado, destinadas a estudiantes avanzados. No requieren una dedicación exclusiva, en tanto suponen que el becario debe completar aún sus estudios de grado, y son pensadas como una suerte de asignación estímulo a la investigación.

- De iniciación, De perfeccionamiento de formación superior: a graduados. El objetivo de la beca la obtención de un título de posgrado, generalmente el de doctor, aunque hay programas que contemplan también el grado de magister.

\section{El caso de la REVIISE}

El contexto de producción académica latinoamericano, como sostiene Beigel (2016) connota un particular dinamismo y atañe al latinoamericanismo como movimiento político y social. En palabras de la autora, Latinoamérica se consolidó como circuito académico durante la década de 1960, sobre todo en las Ciencias Sociales, a partir de un proceso de "regionalización" que se materializó en institutos de investigación universitarios y organismos del estilo de CEPAL, FLACSO, CLACSO, CELADE, ILPES, 
ILADES, DESAL, entre otros. La producción intelectual latinoamericana tuvo una gran expansión junto al auge de la industria editorial en países como México, Argentina y Brasil (Beigel, 2016, p. 12).

La REVIISE, por su parte, fue fundada en el año 2009 y ha publicado un volumen anual, contando en la actualidad con 8 en total. Es la publicación sucesora de los "Cuadernos, Estudios y Traducción", del IISE que tenía como misión difundir las investigaciones del instituto. Estos cuadernos fueron editados durante el periodo 19751990. "A partir de 2011 la edición fue solamente digital. [...] Actualmente la revista ingresa en una nueva etapa adecuando su propuesta editorial bajo la plataforma Open Journal System" (REVIISE, 2016). Nace en un contexto en el cual el MINCYT tenía dos años de antigüedad y el nivel de inversión en ciencia y tecnología presentaba un índice de crecimiento acorde al PBI. Estas situaciones permitieron financiar mayor cantidad de proyectos e incorporar un alto número de jóvenes investigadores a los sistemas de becas e ingresos a carrera de CONICET (Albornoz y Gordon, 2011). Estos nóveles investigadores comenzarían a adquirir roles de importancia explícita en las comisiones de la REVISE.

En este apartado son descriptas las composiciones de los consejos o comités, como el Comité Académico y el Comité Editorial que constituyen a la REVIISE. La misma, se encuentra indexada recientemente en LATINDEX en el $2015 \mathrm{y}$ en otras bases como DIALNET, ERIH PLUS, REDIB, MIAR en el 2016. Por lo tanto, puede clasificarse en la actualidad como integrante de circuitos regionales en Acceso Abierto (Beigel y Salatino, 2015). Aunque es necesario mencionar que en sus primeros volúmenes su inserción en circuitos de difusión respondía a lo propuesto por Beigel y Salatino; a saber: "circuitos locales basados en las publicaciones que no están indexadas, que se publican sólo en papel y por lo general no tienen circulación fuera de límites locales (Beigel y Salatino, 2015, p. 12).

La REVIISE, cuenta con un Comité Académico, que a lo largo de su trayectoria ha variado en su nomenclatura y composición, con el objeto de cumplimentar con las exigencias referentes a las normas de indexación. Las variaciones pueden observarse en la tabla 1:

Tabla 1: Composición de Comité Académico desde 2009 hasta 2016

\begin{tabular}{|l|l|l|}
\hline \multicolumn{1}{|c|}{ Período } & Cantidad de Miembros Internos & $\begin{array}{c}\text { Cantidad de Miembros } \\
\text { Externos }\end{array}$ \\
\hline $2009-2010$ & 16 & -- \\
\hline $2011-2014$ & 15 & -- \\
\hline 2015 & 26 & 21 \\
\hline 2016 & 11 & 15 \\
\hline
\end{tabular}


Como es posible observar, en el 2015 la conformación del Comité Académico de la REVIISE manifestó evidentes modificaciones, presentando una composición de 21 miembros externos al IISE y 26 internos del IISE. Este cambio de estructura fue parte del proceso necesario para gestionar el cumplimiento del protocolo de indexación de LATINDEX, y BINPAR (Bibliografía Nacional de Publicaciones Periódicas Registradas).

El segundo reajuste del Consejo Académico tuvo lugar en el 2016, junto a la indexación en ERIH PLUS; REDIB; MIAR; BASE; DIALNET; ROAD (Naveda, 2016). Para ese número de publicación, el Consejo Académico fue dividido en un Consejo Científico Interno de la Universidad Nacional de San Juan y un Consejo Científico Externo. Como se observa en la tabla 1, el primero conformado por 11 miembros y el segundo por 15. Es necesario aclarar que los miembros del Consejo Científico Externo, suelen poseer un rol simbólico dentro de la institución y no participan de forma regular en presencia de las reuniones pertinentes.

Al igual que la existencia del Comité Académico, la revista desde su inició contó con un Comité Editorial que tiene la función de ser el primer organismo selector de los discursos científicos legítimos para publicar. Sus funciones se atañen a observar y coordinar la adecuación de los artículos postulados a las normativas editoriales. En la tabla 2 muestra sus variaciones desde la fundación de REVIISE.

Tabla 2: Composición de Comité Editorial desde 2009 hasta 2016

\begin{tabular}{|c|c|c|}
\hline Período & Cantidad Miembros Internos & Cantidad Miembros Externos \\
\hline $2009-2012$ & 5 & -- \\
\hline $2013-2014$ & 7 & -- \\
\hline $2015-2016$ & 8 & 1 \\
\hline
\end{tabular}

Este Comité posee una presencia netamente endógena, carácter que no ha sido modificado a partir del ingreso a las indexaciones. Por lo tanto, su estructura ha presentado una dinámica de cambio relativa a los agentes sociales que la intervienen, sin embargo, es uno de los organismos más conservadores en relación a las modificaciones estructurales.

La REVIISE en su trayectoria, ha mantenido la estructura del grado académico de sus autores relativamente estable y completamente conducente con la formación académica de los investigadores del IISE. Se manifiesta, de esta forma, un gran afluente de autores con grados de magister y licenciado/a, y cantidades mínimas de doctores/as, profesores/as, especialistas y el estudiantado. Aunque, desde el año 2014 se observa un leve incremento en la participación de doctores/as como autores en las publicaciones.

Los doctores/as que divulgan en la REVIISE, en su mayoría son investigadores del IISE que han proseguido con su carrera académica. Por lo tanto, se puede 
entender que entre los años 2014 y 2016 no hay doctores externos a este Instituto de Investigación que hayan publicado. Como se observa en la figura 3, es persistente una cierta homogeneidad estructural en relación a la formación de los autores, con un leve ascenso de doctores/as:

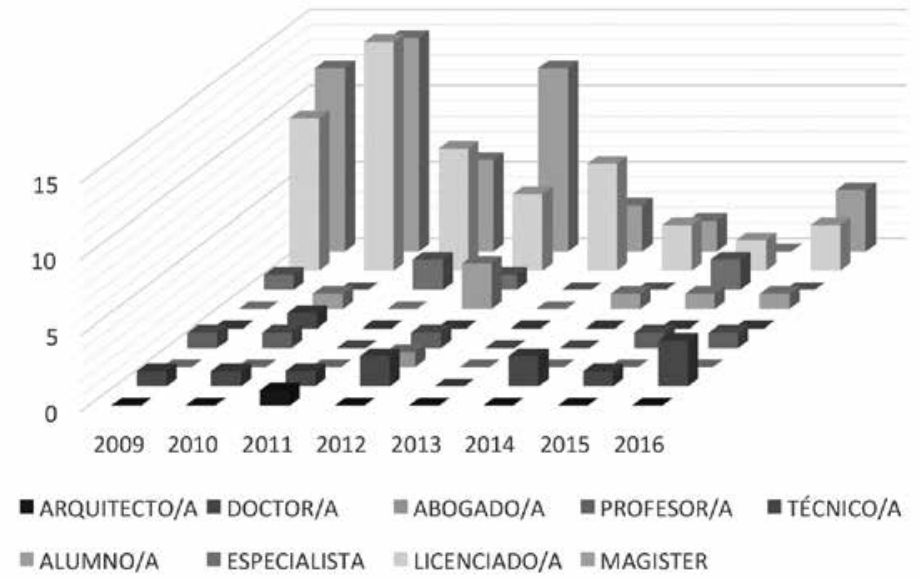

Figura 3: Cantidad de autores por grado académico y año de publicación

Durante los 4 primeros años de publicación de la REVIISE se observa una lógica de difusión netamente endógena. Los artículos publicados tienen directa relación temática con los proyectos de investigación ejecutados en IISE y la cantidad de autores es conducente con los recursos humanos que componen los equipos de investigación. Este comportamiento puede entenderse a partir de la concepción de que REVIISE era, además de un repositorio de las investigaciones internas, un mecanismo de resorte ascendente de legitimación de alumnos/as adscriptos/as y jóvenes investigadores de la FACSO.

Desde el 2013, se produce un considerable descenso de la cantidad de autores en los artículos endógenos, no así de publicaciones; como puede observarse en la figura 4.

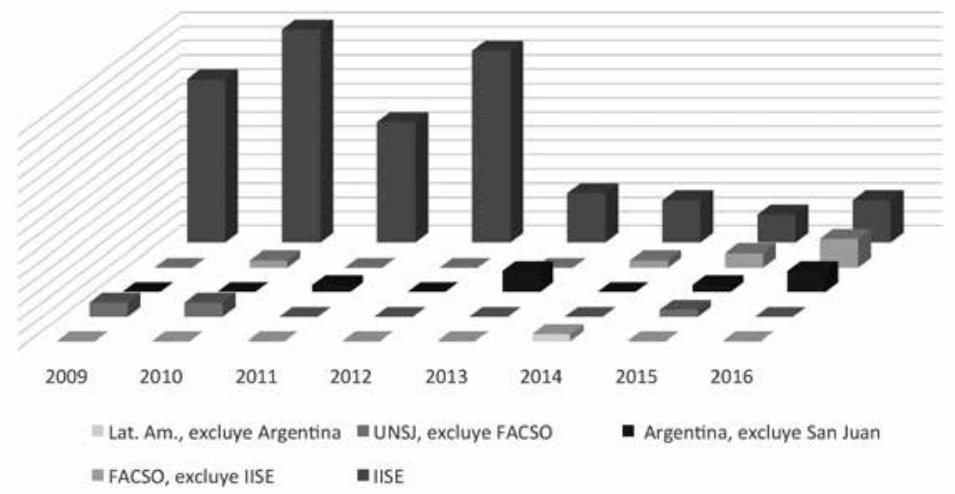

Figura 4: Autores por año de publicación de la REVIISE, según pertenencia académica 
La figura anterior demuestra que los autores cuya pertenencia académica es el IISE son quienes representan la mayor reducción. Paralelamente, se percibe el inicio de una tendencia al ascenso de quienes tienen sede en FACSO, sin incluir al IISE y quienes tienen sede en algún Instituto o Universidad de Argentina, sin incluir a la UNSJ o la UCC. Los últimos dos años muestran una tendencia de mayor heterogeneidad autoral, en detrimento de los primeros 4 años que presentan una hegemonía netamente endógena de los autores evaluados como pertinentes de publicar.

Entre 2013-2016, los artículos difundidos por REVIISE ya no presentan una estructura isomórfica a la de un Informe final devenido en ponencia y posterior artículo en la cual participaba la totalidad de un equipo de investigación. Sino, son textualidades que continúan recorriendo temáticas de investigación de IISE, pero con una orientación individualizada. Comienza a observarse la emergencia de subcomisiones en los equipos que se encargan de la producción de artículos para publicar. Por esa razón, en REVIISE se torna presente un fenómeno de redundancia autoral endógena. Se observa que la presencia de autores que, en conjunto o en solitario, han logrado superar más de una vez los mecanismos de evaluación, consiguiendo la publicación, siendo todos miembros del IISE. La tabla 3, presenta la distribución de los autores según su cantidad de artículos publicados en REVIISE:

Tabla 3: Cantidad de autores por cantidad de publicaciones en REVIISE

\begin{tabular}{|l|l|l|}
\hline \multicolumn{1}{|c|}{ Tipo de Autor } & \multicolumn{1}{|c|}{ Cantidad } & \multicolumn{1}{c|}{ Relación porcentual } \\
\hline Autor que ha publicado una vez & 80 & $74,7 \%$ \\
\hline Autor que ha publicado dos veces & 22 & $20,37 \%$ \\
\hline Autor que ha publicado tres veces & 6 & $5,5 \%$ \\
\hline
\end{tabular}

Como se mencionó más arriba, todos los autores que tienen más de una publicación en la REVIISE han sido becarios, adscriptos o miembros de algún equipo de investigación del IISE en el mismo período en el que sus artículos fueron publicados. Además, la hegemonía endógena se presenta en la categoría de quienes han publicado una vez. De los 80 autores con un único artículo, 58 pertenecen al IISE, 8 integran el GEITS (Gabinete de Estudios e Investigación de Trabajo Social de la FACSO). 5 de facultades de la UNSJ, -sin incluir a FACSO-: Facultad de Filosofía, Humanidades y Artes, Facultad de Arquitectura y la Facultad de Ciencias Exactas, Físicas y Naturales. Asimismo, hay autores externos, cuya procedencia es la Universidad Nacional de Cuyo, la Universidad del Nordeste, El Instituto Gino Germani y la Universidad de Buenos Aires. Y por último el único autor fuera de Argentina pertenece a la Universidad Autónoma de Guerra, México.

Si los artículos publicados se ordenan según el criterio de Áreas de Investigación que posee el IISE, se puede precisar que, de 61 artículos 30 responden a la categoría Estado, Gobierno y Sociedad, 11 a Socioeconómica, Regional Social y Urbana y 9 a 
Educación, Cultura y Sociedad. Existe un explícito diferencial entre las publicaciones en torno a la Sociología en relación con las otras áreas de investigación. Sin embargo, la variable temporal no influye de manera determinante en relación con las temáticas publicadas como sugiere la figura 5 .

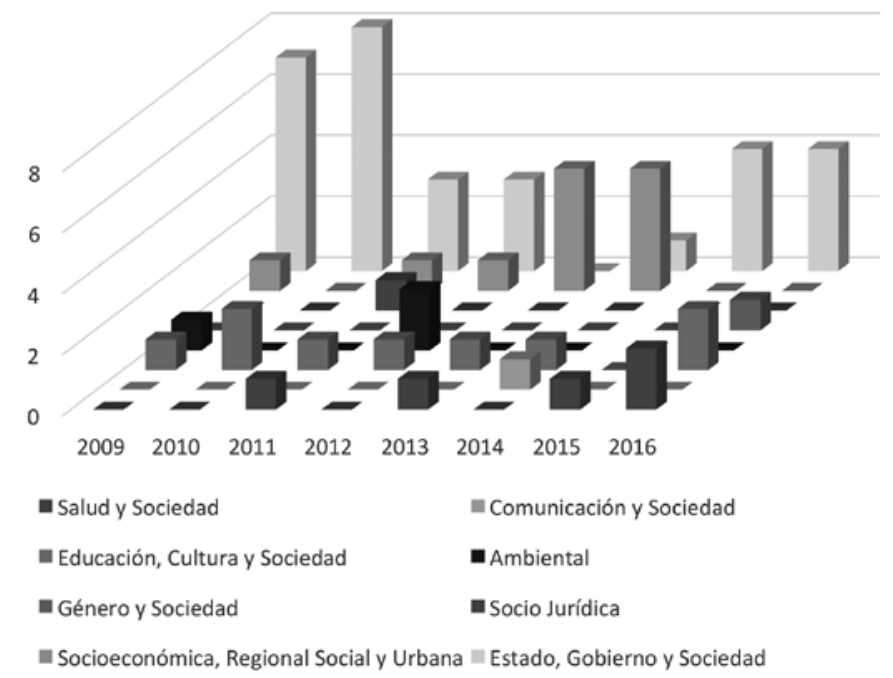

Figura 5: Área investigación del tema de artículo según año de Publicación

\section{Conclusiones}

Describir las características de quienes producen el conocimiento científico, y las formas en que se conforman sus estrategias de producción y/o difusión de conocimientos dentro de su Instituto es de relevante interés para iniciar los estudios sobre las capacidades de investigación.

En este caso como se ha podido observar se toma como punto de referencia para el análisis la REVIISE que durante sus primeros cinco años de existencia mantuvo una estructura representada por relativas estabilidades en torno a sus Comité Científico/ Académico y Editoriales. De la misma forma, sus lógicas de evaluación, publicación y difusión no sólo fueron tributarias de su antecesor «Cuadernos, Estudios y Traducción", sino que también profundizaron mecanismos de reproducción de prácticas netamente endógenas. En ese lustro, el 9o\% de los autores eran miembros del IISE.

Sin embargo, el 2014 funciona como un año clivaje, en el cual la matrícula de autores externos al IISE, como se observó en este trabajo, ascendió. Y en 2015 al restructurarse el Comité Académico se dio lugar a una gran cantidad de becarios CONICET. Esta situación presenta rasgos similares por lo propuesto por Bourdieu al sostener que "los cambios observados en la política editorial de las diferentes editoriales pueden, así, 
estar relacionado a cambios de la posición que ellas ocupan en el campo". (Bourdieu, 1997, p. 225). Aunque es posible percibir como disposiciones de cambios que intentan modificar de manera exprofeso la posición que ocupa la REVIISE en el circuito de las Revistas Académicas locales.

Las renovaciones de agentes sociales en comitivas editoriales, la diversificación y externalización (de forma simbólica) del Consejo Académico vislumbran las intenciones de romper con la endogamia que ha marcado a esta publicación. Uno de los factores que puede haber llevado a esas decisiones es la concientización" del IISE en la última década. Esto ha motivado a reforzar las estrategias para la sostener el ingreso y mantención de los recientes investigadores afiliados a CONICET, dentro del Consejo. Una de ellas puede ser la indexación de la REVIISE con el objeto de lograr el cumplimiento de algunos de los requisitos para el ingreso a carrera en CONICET. "La encuesta que realizamos en las comisiones evaluadoras del CONICET (Argentina) demuestra que en estas disciplinas [ciencias sociales y humanidades] también opera el reemplazo de la evaluación de la calidad de los trabajos por la evaluación de la indexación de las revistas" ( Beigel, 2016, p. 13). Por ese motivo puede entenderse que el interés por la indexación e intentos de transnacionalización (Beigel y Salatino, 2015) se encuentra atravesado principalmente por una necesidad de generar un recurso de afianzamiento en el campo científico nacional de los investigadores del IISE.

Se puede concluir que la REVIISE se encuentra en un momento de cambio de posiciones dentro del campo de las Revistas Científicas Argentinas. Esta situación lleva a sus agentes a practicar un ejercicio de búsqueda de legitimación trascendente de lo local, entrando en el interjuego de negociación de capital tanto simbólico como científico, con los repositorios y bases latinoamericanas como con la comunidad científica nacional y de América Latina. Este comportamiento es acorde al sostenido por Beigel y Salatino (2015) ya que "en Argentina, los repositorios y bases de registros latinoamericanos (SciELO, Latindex-C, RedALyC), cubren prácticamente la totalidad las revistas indexadas." (Beigel y Salatino, 2015, p. 21). Consecuentemente, se puede pensar que la búsqueda actual de la REVIISE consiste en una nueva posición dentro de este campo que funcione de modo de andamiaje o acompañamiento a las posiciones que los recursos humanos del IISE comienzan a ocupar en el campo científico argentino. De modo que se busca una transnacionalización e indexación para adecuarse a las nuevas prácticas endógenas que afloran en este instituto.

\section{Lista de referencias}

Albornoz, M., \& Gordon, A. (2011). La política de ciencia y tecnología en Argentina desde la recuperación de la democracia (1983 - 2009). En M., Albornoz y J., Sebastián (Eds.) Trayectorias de las políticas científicas y universitarias de Argentina y España. Madrid: CSIC, 
Algañaraz, V. (2014). Reestructuración universitaria en clave autoritaria: politica y accionar de los rectores de la Universidad Nacional de San Juan durante la última dictadura militar (1976-1983). PolHis 7(4), 236-255.

Altbach, P. (2009). Educación Superior Comparada: el conocimiento, la universidad y el desarrollo. Buenos Aires: Universidad de Palermo.

Beigel, F. (2016). El nuevo carácter de la dependencia intelectual. Cuestiones de Sociología, 14, e004. Recuperado de

Beigel, F., \& Salatino, M. (2015). Circuitos segmentados de consagración académica: las revistas de Ciencias Sociales y Humanas en la Argentina. Información, cultura y sociedad /32

Bourdieu, P. (1997). Razones prácticas. Sobre la teoría de la acción. Barcelona, España. Anagrama.

Bourdieu, P. (2008). Homo Académicus. Buenos Aires: Edit. Siglo veintiuno.

Bourdieu, P. y Passeron, J. (2003) Los herederos. Los estudiantes y la cultura. Buenos Aires: Edit. Siglo veintiuno.

Hall, S. (2013). Discurso y Poder. Huancayo. Perú. http://www.cuestionessociologia. fahce.unlp.edu.ar/article/view/CSn14ao4

Medina, P. (2000). ¿Eres maestro normalista y/o universitario? La docencia cuestionada. México. Ciesas.

Naveda, A. (2016). Informe de indexación de la REVIISE una revista de Ciencias Sociales y Humanas del Instituto de Investigaciones Socio-Económica. Recuperado de http:// www.ojs.unsj.edu.ar/index.php/reviise/

REVIISE (2016). Historia acerca de la revista de Ciencias Sociales y Humanas del Instituto de Investigaciones Socio-Económicas. Recuperado de http://www.ojs.unsj.edu. ar/index.php/reviise/

UNSJ. (1994). La Universidad Nacional de San Juan. Su historia y proyección regional. Tomo II. San Juan: EFU,

UNSJ. (2013). Los cinco elementos. Génesis de las unidades académicas de la UNSJ. Edición especial: 40 años de la Universidad Nacional de San Juan, N 7(6). 